\title{
Uma Abordagem Evolucionária Multiobjetiva para a Alocação de Unidades de Comunicação em Redes Veiculares para a Distribuição de Streaming
}

\author{
Leonardo A. A. Pereira ${ }^{1}$, Mateus C. Luciano ${ }^{1}$, Larissa L. Mariz ${ }^{1}$ \\ Bruna V. Ramos $^{1}$, Cristiano G. Pitangui ${ }^{1}$, Cristiano M. Silva ${ }^{1}$ \\ ${ }^{1}$ Universidade Federal de São João Del-Rei - Minas Gerais - Brasil \\ leonardoaapereira@gmail.com, mateuscalais@outlook.com, \\ larissa.lopes30@hotmail.com, brunavilarino21@gmail.com \\ \{pitangui.cristiano, cristiano\}eufsj.edu.br
}

\begin{abstract}
In this work we consider the deployment of roadside units (RSUs) for communication in vehicular networks. Basically, we intend to promote regular vehicle-to-infrastructure encounters allowing vehicles to continuously receive media from roadside units while driving. In terms of QoS parameters, we consider (a) the share of vehicles demanding the streaming service, (b) the data transfer rate from roadside units to vehicles, and $(c)$ the data consumption rate inside vehicles. After formally formulating the problem, we present the Sigma-N deployment strategy using the NSGA-II evolutionary multi-objective algorithm. We compare Sigma-N to the intuitive strategy of placing roadside units at the densest locations of the road network. The results show that Sigma-N requires less RSUs to achieve the same coverage provided by the densest locations strategy, while providing similar QoS.
\end{abstract}

Resumo. Este trabalho considera a deposição de unidades de comunicação (RSUs) em redes veiculares. Basicamente, têm-se o objetivo de promover encontros regulares de veículos com a infraestrutura, permitindo que os veículos continuem recebendo mídia da infraestrutura durante sua locomoção. Em termos de parâmetros de QoS, são considerados: (a) a fração de veículos que receberá a transmissão de mídia, (b) a taxa de recepção de dados da infraestrutura para os veículos, e (c) a taxa de consumo de dados dentro dos veículos. Após a formulação do problema, este trabalho apresenta a estratégia de deposição Sigma-N que utiliza o algoritmo evolucionário multiobjetivo NSGA-II. Sigma-N é comparada com a estratégia intuitiva de se alocar unidades de comunicação em pontos de maior concentração de fluxo. Os resultados mostram que Sigma-N requer menos RSUs para atingir a mesma cobertura, enquanto fornece similar qualidade de serviço.

\section{Introdução}

A grande disseminação de dispositivos móveis na população mundial traz perspectivas sem precedentes. O mundo conta com mais de sete bilhões de smartphones conectados à Internet, muitos deles equipados com sensores de última geração provendo dados em tempo real, capazes de subsidiar um sistema de vigilância mundial. Ao mesmo tempo, 
os grandes centros urbanos passam por um período de aumento de sua população (maior expectativa de vida, movimentos migratórios, etc), impondo grandes desafios para os governos em termos de saúde pública, segurança e mobilidade urbana.

De acordo com Van Audenhove et al. [2014], "53\% da população mundial vive hoje em cidades. A previsão é que em 2050 tenhamos 67\% da população vivendo em cidades. Hoje, $64 \%$ de todos os deslocamentos se dão em ambientes urbanos, e espera-se que o total de kilômetros viajados pela população dentro de ambientes urbanos triplique até 2050". Assim, novas soluções são fundamentais para que a sociedade consiga lidar com essa impressionante demanda por mobilidade. Em fato, a mobilidade urbana é um problema multifacetado que exige a adoção de diversas medidas sincronizadas, incluindo, também, um melhor uso da infraestrutura viária atualmente disponível, o que pode ser obtido pela integração das chamadas Tecnologias de Informação e Comunicação no desenvolvimento de Sistemas Inteligentes de Transportes (SIT) [Silva et al., 2015].

Nesse sentido, desenvolver a habilidade de gerenciar redes veiculares [Silva and Meira Jr, 2015c] é um passo fundamental para que se consiga ter essas redes implantadas em larga escala subsidiando a operação de SIT [Silva et al., 2017a]. Um dos desafios relaciona-se à alta mobilidade dos veículos, que impõe um novo padrão de uso da rede. Veículos não demandam cobertura contínua [Silva and Meira Jr, 2015a]. Ao invés disso, espera-se que a comunicação se dê por sua travessia em ilhas de cobertura estrategicamente posicionadas ao longo da malha viária. Através da disponibilização de uma infraestrutura de comunicação (aqui chamada de unidades de comunicação, do inglês roadside units ou RSUs), pode-se criar uma rede veicular que permita a disseminação de dados.

Este trabalho apresenta uma solução para a alocação de RSUs em redes veiculares para a distribuição de streaming, onde a regularidade de contatos entre veículos e a infraestrutura é fundamental. De forma complementar, tais aplicações também demandam um controle assertivo sobre o intervalo entre conexões, de forma que os veículos sempre mantenham um buffer de dados suficiente para que a mídia seja reproduzida sem interrupção até o próximo contato com a infraestrutura (onde novos dados serão recebidos).

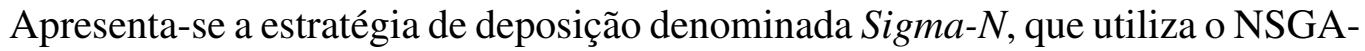
II (Non-dominated Sorting Genetic Algorithm) [Deb et al., 2002] para realizar a deposição de unidades de comunicação ao longo de uma malha viária de topologia complexa, criando ilhas de coberturas que serão atravessadas por veículos. De forma geral, o NSGA-II é uma extensão do Algoritmo Genérico (GA) [Goldberg, 1989] utilizado na solução de problemas de otimização multiobjetivo. Uma dada deposição $\Sigma_{N}\left(\begin{array}{l}\tau \\ \rho\end{array}\right)\left(\begin{array}{l}v_{d} \\ v_{c}\end{array}\right)$ garante que $\rho$ porcento dos veículos passam por ilhas de cobertura (zonas com RSUs) em intervalos de tempo não-maiores que $\tau$ segundos, e que estes veículos mantêm seu buffer com dados disponíveis, tendo como restrição a velocidade de download de dados das RSUs $\left(v_{d}\right)$, bem como a velocidade de consumo de dados dentro do veículo $\left(v_{c}\right)$.

A estratégia proposta é avaliada em termos do investimento necessário para a montagem da infraestrutura, bem como da qualidade de serviço ofertada pela rede. A comparação se dá com a estratégia intuitiva de se alocar unidades de comunicação nas áreas de maior fluxo de veículos. Os resultados demonstram que Sigma-N é eficaz em prover uma cobertura semelhante mediante uma redução no investimento de, até, $40 \%$ para a montagem da rede. 
O restante desse documento encontra-se organizado da seguinte forma. A seção 2 apresenta os trabalhos relacionados. A seção 3 formula o problema da Deposição Sigma. A seção 4 apresenta como a Deposição Sigma pode ser abordada como um problema de otimização multiobjetivo. A seção 5 apresenta a estratégia Sigma-N. A seção 6 apresenta a estratégia utilizada como baseline (deposição de unidades de comunicação nas áreas de maior densidade de veículos). A seção 7 discute os resultados obtidos, enquanto que a seção 8 conclui o trabalho e apresenta algumas possibilidades de pesquisas futuras.

\section{Trabalhos Relacionados}

A deposição de infraestrutura para redes veiculares é estudada sobre diferentes pontos de vista. Um survey sobre o tema é apresentado em [Silva et al., 2017a]. Estudos analíticos concentram-se, tipicamente, na definição de limites teóricos máximos para a transmissão de dados, seja em termos da quantidade de dados disseminados, ou da latência. Como exemplo, Zheng et al. [2010] apresentam a avaliação de uma estratégia de implantação de infraestrutura através do conceito de oportunidade de contato. Já Lee and Kim [2010] propõem uma heurística para a implantação de infraestrutura que busca ampliar a conectividade dos veículos e reduzir o intervalo sem conexões. Por sua vez, Nekoui et al. [2008] propõem uma técnica baseada na definição de capacidade de transporte.

A probabilidade de contato também é considerada. Zheng et al. [2010] apresentam a avaliação da estratégia de implantação considerando a oportunidade de contato. A métrica mensura a fração da distância (ou tempo) que o veículo está em contato com a infraestrutura, enquanto que Lee and Kim [2010] propõem uma heurística gulosa para alocar a infraestrutura com o objetivo de melhorar a conectividade entre veículos, ao mesmo tempo em que reduzem suas desconexões, e cada interseção é considerada como uma potencial posição para uma unidade de comunicação.

Xie et al. [2013] abordam o problema de alocação de unidades de comunicação assumindo conhecimento prévio da trajetória de cada veículo. Baseando-se em dados históricos, os autores propõem um modelo probabilístico para inferir as localizações das unidades de comunicação. Já, Chi et al. [2013] consideram uma estratégia que distribui as unidades de comunicação tão equilibradamente quanto possível. No entanto, os autores comparam seus resultados com uma estratégia de alocação aleatória que incorre em um desempenho muito baixo. De forma complementar, Liu et al. [2013] propõem uma estratégia de alocação de unidades de comunicação para a transferência de arquivos em redes veiculares modelando o encontro entre veículos e unidades de comunicação como uma cadeia da Markov homogênea de tempo contínuo.

Em termos de estratégias geométricas, Cheng et al. [2013] propõem uma heurística para solucionar o problema da máxima cobertura, enquanto que Patil and Gokhale [2013] empregam diagramas de Voronoi. Já em termos de modelos de otimização, Aslam et al. [2012] usam Programação Inteira Binária para o problema de alocação de infraestrutura eliminando vias de pouco tráfego.

Em termos de heurísticas, Trullols et al. [2010] propõem a modelagem de alocação de RSUs como um problema de máxima cobertura, assumindo-se a premissa de conhecimento prévio das trajetórias dos veículos. Esses estudos são evoluídos para remover essa restrição no trabalho [Silva et al., 2016b], que se baseia na predição Markoviana do fluxo.

Alguns estudos abordam o problema de distribuição de conteúdo no contexto de 
redes veiculares. Gossa et al. [2008] propõem uma solução de replicação de dados proativa para selecionar veículos como réplicas. Por outro lado, Li et al. [2015] replica o conteúdo em unidades de comunicação com base na análise do rastro de mobilidade real, enquanto que Silva et al. [2017b] discutem uma rede veicular de distribuição de conteúdos, a chamada Vehicular Content Delivery Network.

Já o gerenciamento da rede veicular e definição de métricas de desempenho são discutidos pelo nosso grupo de pesquisa nos trabalhos [Silva and Meira Jr, 2015c; Silva et al., 2016a]. Em [Silva and Meira Jr, 2016], apresentamos uma arquitetura híbrida envolvendo unidades de comunicação móveis e estacionárias para lidar com mudanças no padrão de fluxo. Em termos gerais, caminhamos no sentido de definir um conjunto de métricas capaz de permitir o planejamento e gerenciamento de redes veiculares de forma que provedores do serviço tornem-se capazes de operacionalizar essas redes.

Diferentemente dessas abordagens, a estratégia aqui proposta considera o tempo entre contatos e o controle da carga de dados do veículos (dados em buffer) para identificar os melhores locais para a implantação das RSUs, permitindo a elaboração de estratégias sofisticadas para a entrega de conteúdos em redes veiculares.

\section{Formulação do Problema: A Deposição Sigma}

O planejamento de redes veiculares infraestruturadas requer a definição da localização física de cada unidade de comunicação, criando-se ilhas de cobertura estrategicamente posicionadas para alimentar os veículos com dados. Esse trabalho volta sua atenção para o planejamento dessa infraestrutura no contexto de disseminação de streaming. O objetivo é que veículos experimentem contatos regulares com a infraestrutura, de forma a permitir que mídia seja continuamente enviada aos veículos. De forma similar, também pretendese controlar o intervalo entre contatos, garantindo que veículos não fiquem muito tempo sem receber conteúdo, permitindo que os ocupantes do veículo sempre tenham dados de streaming disponíveis. Nesse sentido, a Deposição Sigma $\Sigma_{N}\left(\begin{array}{l}\tau \\ \rho\end{array}\right)\left(\begin{array}{l}v_{d} \\ v_{c}\end{array}\right)$ é proposta como uma métrica de qualidade de rede baseada em quatro parâmetros: $\tau, \rho, v_{c}, v_{d}$.

O parâmetro $\tau$ especifica o tempo máximo entre dois contatos consecutivos entre um veículo e a infraestrutura. $\mathrm{O}$ parâmetro $v_{c}$ indica a taxa de consumo da mídia dentro dos veículos. O parâmetro $v_{d}$ indica a taxa com que dados são baixados das unidades de comunicação para os veículos. Já o parâmetro $\rho$ indica a quantidade de veículos que devem ser capazes de reproduzir a mídia sem interrupções durante toda a sua viagem. Formalmente:

Definição 1 (Deposição Sigma: $\Sigma_{N}\left(\begin{array}{l}\tau \\ \rho\end{array}\right)\left(\begin{array}{l}v_{d} \\ v_{c}\end{array}\right)$ ). Seja $R$ o conjunto das vias de uma malha viária. Seja $V$ o conjunto de veículos que viaja em $R$. Cada veículo $v_{i} \in V$ recebe dados das unidades de comunicação à taxa de $v_{d}$ bits por segundo que são armazenados dentro de um buffer interno ao veículo. De forma similar, cada veículo vi consome dados de seu buffer interno à taxa de $v_{c}$ bits por segundo. Uma dada distribuição de unidades de comunicação sobre $R$ é considerada uma Deposição Sigma $\Sigma_{N}\left(\begin{array}{l}\tau \\ \rho\end{array}\right)\left(\begin{array}{l}v_{d} \\ v_{c}\end{array}\right)$ quando um subconjunto de veículos $V^{\prime} \subseteq V$ consegue realizar seu trajeto sempre mantendo seu buffer interno com tamanho $>0$ (ou seja, a quantidade de dados recebidos foi maior que a quantidade de dados consumidos). Além disso, esses veículos devem encontrar a infraestrutura em intervalos nunca maiores do que $\tau$ segundos, sendo que $\frac{\left|V^{\prime}\right|}{|V|}=\rho$. 
Para resolver a Deposição Sigma, assume-se o particionamento da área urbana em um conjunto de $\psi \times \psi$ células urbanas de mesmo tamanho. As células urbanas podem ter tamanhos arbitrários, a fim de atender às necessidades de projeto da rede. Quando se exige maior/menor precisão, simplesmente aumenta-se/diminui-se o número de células urbanas que cobrem a malha rodoviária. Particionando-se a malha rodoviária, diminui-se o número de locais a serem avaliados para receberem unidades de comunicação. Tal particionamento acarreta, portanto, uma redução do esforço computacional para a obtenção das melhores localidades. As figuras 1(a)-1(d) exemplificam esse processo.

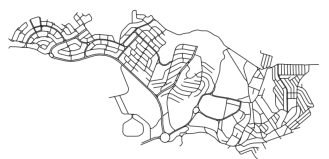

(a) Malha Rodoviária

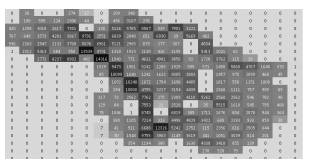

(b) Partição 20 x 20

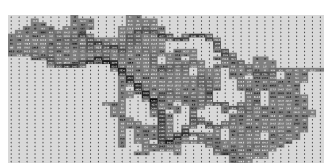

(c) Partição 40 x 40

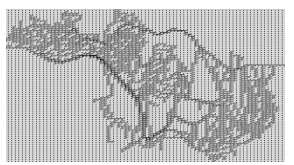

(d) Partição 80 x 80

Figura 1. Particionamentos da Malha Viária em Grides de Diferentes Resoluções.

O tempo entre contatos $\tau$ regula a velocidade com que os dados são disseminados. Uma redução no valor de $\tau$ acarreta redução na quantidade de unidades de comunicação utilizadas, porém diminui a precisão de controle da rede, visto que as informações irão levar mais tempo para atingir os usuários. A taxa de download $v_{d}$ (velocidade cujos dados são baixados) e a taxa de consumo $v_{c}$ (velocidade na qual dados são consumidos) controlam a carga disponível no buffer interno do veículo. Se um veículo possui carga positiva, significa que ele possui mídia disponível para ser consumida.

A diferença entre a velocidade de download e a velocidade de consumo é chamada de velocidade de recarga. Para que se tenha uma configuração válida, é necessário que a velocidade de download seja sempre maior que a velocidade de consumo, caso contrário a velocidade de recarga será negativa. Desse modo, quando um veículo está dentro da área de cobertura de uma RSU, ele está ganhando mais carga do que consome, pois $v_{d}>v_{c}$, ou seja, está recarregando. Quando está fora da área de cobertura, ele está perdendo carga.

\section{A Deposição Sigma como Problema de Otimização Multiobjetivo}

Otimização é o processo de identificação da melhor solução entre um conjunto de alternativas [Miettinen, 1999]. De forma geral, a otimização de um único objetivo emprega um único critério (objetivo) para identificar a melhor solução entre um conjunto de soluções. Por sua vez, a otimização multiobjetivo emprega dois ou mais critérios (objetivos) com esta finalidade. Uma vez que múltiplos objetivos podem ser conflitantes, i.e, a melhora de um pode levar a deterioração de outro(s), geralmente não existe solução ótima única para problemas de otimização multiobjetivo. Um problema de otimização composto por $k$ objetivos pode ser formalizado como apresentado na definição 2 .

Definição 2 (Problema de Otimização Multiobjetivo). Dado um vetor de variáveis de decisão com dimensão $n, x=\left\{x_{1}, \ldots, x_{n}\right\}$ no espaço de busca $X$, objetiva-se encontrar $x^{*} \in X$ que minimiza, simultaneamente, as $k$ funções objetivo $f\left(x^{*}\right)=\left\{f_{1}\left(x^{*}\right), \ldots, f_{k}\left(x^{*}\right)\right\}$. Assim, o modelo geral de um problema de otimização multiobjetivo para o caso de minimização é dado por:

$$
\operatorname{Min} f\left(x^{*}\right)=\left\{f_{1}\left(x^{*}\right), \ldots, f_{k}\left(x^{*}\right)\right\} \text { s. } a x \in X, \text { onde: } f_{i}: R^{n} \rightarrow R, \text { e } i \geq 2 .
$$


O conjunto das soluções não-dominadas em $X$ é chamado de conjunto Paretoótimo, e a imagem de um determinado conjunto Pareto-ótimo, no espaço dos valores dos objetivos, é chamada de fronteira de Pareto [Konak et al., 2006]. O conceito de dominância é apresentado na definição 3, enquanto que a solução Pareto-ótima é apresentada na definição 4.

Definição 3 (Dominância). Uma solução $x$ domina a solução y, se e somente se $f_{i}(x) \leq f_{i}(y)$ para $i=1$, ..., k e $f_{i}(x)<f_{i}(y)$ para, ao menos, uma função objetivo $j$.

Definição 4 (Solução Pareto-ótima ou não-dominada). Uma solução é dita Pareto-ótima, ou não-dominada, se não for dominada por nenhuma outra solução no espaço de busca. Uma solução Pareto-ótima não pode ser melhorada com relação a qualquer objetivo sem que exista piora para pelo menos algum outro objetivo.

Considerando-se o problema de Deposição Sigma, percebem-se dois objetivos conflitantes, uma vez que almeja-se a redução do número de unidades de comunicação alocadas, como forma de reduzir o custo da rede veicular, ao mesmo tempo em que se deseja aumentar o número de veículos tendo oportunidades de contato com a infraestrutura de comunicação. Tal problema deve, portanto, ser solucionado buscando-se minimizar o número de unidades de comunicação utilizadas, ao mesmo tempo em que busca-se maximizar o número de veículos recebendo a Cobertura Sigma.

Definição 5 (Cobertura Sigma). A Cobertura Sigma refere-se a depositar unidades de comunicação ao longo da malha viária de forma que $\rho$ porcento dos veículos atravessem ilhas de cobertura em intervalos não-maiores que $\tau$ segundos, recebendo conteúdo das unidades de comunicação à taxa de $v_{d}$ bits por segundo e consumindo esses dados, continuamente, à taxa de $v_{c}$ bits por segundo, sem nunca zerar sua carga, i.e., seu buffer interno de armazenamento.

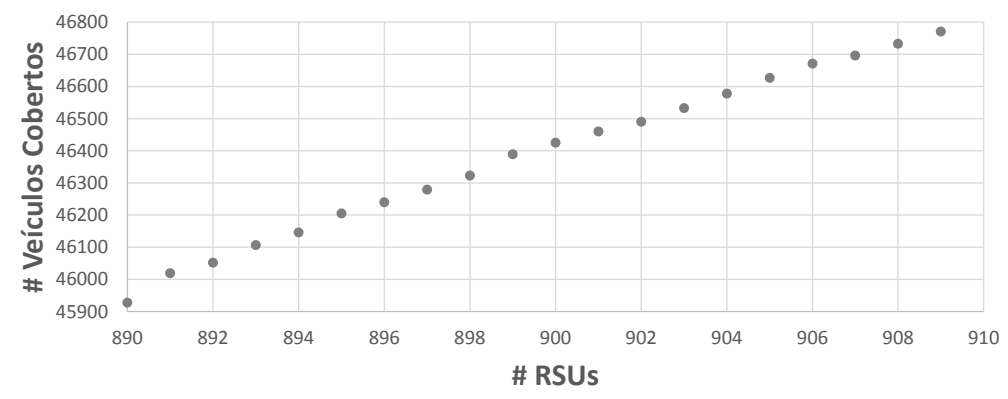

\section{Figura 2. Fronteira de Pareto: Número de Veículos Cobertos versus Número de Unidades de Comunicação Depositadas.}

Neste sentido, a otimização multiobjetivo busca um compromisso entre os vários objetivos expresso pela fronteira de Pareto. Caso seja possível otimizar todos os objetivos, a fronteira de Pareto será constituída por apenas um ponto, ou seja, pela solução ótima. Caso contrário, a fronteira de Pareto será constituída por diversas soluções. Considerando a minimização do número de unidades de comunicação e a maximização da quantidade de veículos com Cobertura Sigma como objetivos, para uma determinada instância do problema de Deposição Sigma, a figura 2 representa uma fronteira de Pareto. Note que não é possível melhorar um dos objetivos sem que se piore o outro. 


\section{Estratégia de Deposição Sigma- $N$}

Com base nos conceitos já apresentados, pode-se apresentar a estratégia de deposição Sigma-N. Essa estratégia utiliza o NSGA-II [Deb et al., 2002] para realizar a deposição de RSUs. O NSGA-II simula o processo evolutivo de indivíduos (soluções) pela utilização de operadores de busca que incluem a seleção dos indivíduos mais adaptados, a recombinação (crossover) entre indivíduos, e mutação. A implementação do algoritmo NSGA-II utilizado nesse trabalho faz parte do MOEA Framework ${ }^{1}$, uma biblioteca Java gratuita e de código aberto voltada para aplicações de otimização multiobjetivo.

A escolha do NSGA-II se justifica uma vez que ele é um dos algoritmos evolucionários mais utilizados em otimizações multiobjetivo, sendo bastante eficiente na obtenção de bons resultados em vários tipos de problemas ao mesmo tempo em que influencia a criação/modificação de vários outros métodos de otimização multiobjetivo [Ye et al., 2015]. O NSGA-II presente no MOEA foi adaptado para lidar com esse problema.

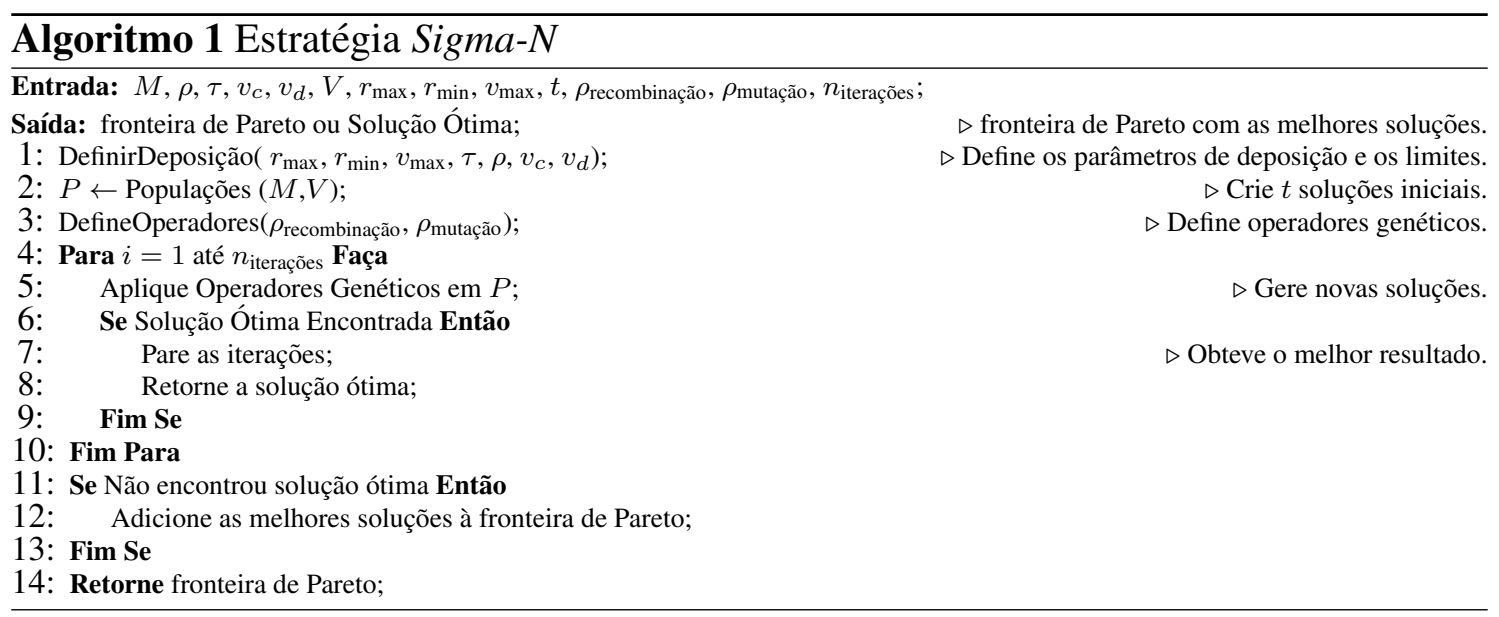

O algoritmo 1 apresenta a Deposição Sigma-N. Os parâmetros de entrada são apresentados na tabela 1. Repare que a terceira coluna indica sua natureza (se o parâmetro surge do problema em si, ou é necessário para aplicação da técnica de otimização).

Tabela 1. Parâmetros de Entrada de Sigma-N

\begin{tabular}{|l|l|l|}
\hline Parâmetro & Descrição & Natureza \\
\hline$M$ & matriz bidimensional representando a malha viária particionada & Problema \\
$\rho$ & fração de veículos que deve receber Cobertura Sigma & Problema \\
$\tau$ & tempo máximo entre contatos de veículos com a infraestrutura & Problema \\
$v_{c}$ & velocidade de consumo de dados & Problema \\
$v_{d}$ & velocidade de download de dados & Problema \\
$V$ & conjunto de veículos e suas trajetórias & Problema \\
$r_{\max }$ & número máximo de RSUs que podem ser depositadas durante a otimização & Otimização \\
$r_{\min }$ & número mínimo de RSUs que o algoritmo pode usar durante a otimização & Otimização \\
$v_{\max }$ & número máximo de veículos que se deseja cobrir & Otimização \\
$t_{\text {população }}$ & número de soluções que o algoritmo trabalhará durante o processo de busca & Otimização \\
$\rho_{\text {recombinação }}$ & taxa de recombinação: probabilidade de recombinação (crossover) & Otimização \\
$\rho_{\text {mutação }}$ & taxa de mutação: taxa (probabilidade) de mutação & Otimização \\
$n_{\text {iterações }}$ & número de iterações: ciclos que o algoritmo executará & Otimização \\
\hline
\end{tabular}

\footnotetext{
${ }^{1}$ http://moeaframework.org/
} 
O algoritmo1 inicia configurando os parâmetros de deposição que serão utilizados para realizar a deposição inicial. Obter boas soluções iniciais é importante para reduzir o tempo de convergência do algoritmo. Repare que um limite de interações é definido através do parâmetro $n_{\text {iterações }}$. Caso o algoritmo inicie com soluções de baixa qualidade, isso pode comprometer os resultados pelo esgotamento da quantidade de interações sem a obtenção de soluções de qualidade.

A população inicial é criada alocando-se as unidades de comunicação de acordo com a estratégia de alocação de RSUs Sigma- $\phi$ [Pereira et al., 2017]. De forma resumida, Sigma- $\phi$ processa a rota de todos os veículos e atribui um score às células urbanas, de acordo com sua contribuição em termos de cobertura de veículos. Iterativamente, a estratégia seleciona a célula urbana com maior contribuição numa abordagem gulosa. Esse processo é repetido até que $\rho$ porcento dos veículos estejam cobertos. Após a escolha de uma solução viável, aplica-se um processo de busca local que objetiva encontrar um ponto de máximo local. Sigma- $\phi$ é discutida em detalhes em [Pereira et al., 2017].

Após geradas $t$ soluções iniciais para o NSGA-II, são configurados os operadores genéticos, e os limites do problema em questão. O NSGA-II realizará, então, $n_{\text {iterações }}$ iterações de modo a encontrar uma fronteira de Pareto. Dentro de cada iteração, os operadores genéticos são aplicados de acordo com os parâmetros $\rho_{\text {recombinação }}$ e $\rho_{\text {mutação na }}$ população (conjunto de soluções em $P$ ), buscando seu aprimoramento. Uma dada solução é considerada viável quando $\rho$ porcento dos veículos apresentam: (a) tempo entre contatos menor ou igual que $\tau$, e (b) carga mantêm-se acima de zero durante toda sua trajetória.

\section{Baseline: Alocando Unidades de Comunicação nos Pontos de Maior Concentração de Veículos (Estratégia DL)}

Com o objetivo de comparar o desempenho da estratégia Sigma- $N$, utiliza-se a estratégia DL (Densest Locations), que consiste em alocar as unidades de comunicação nos pontos de maior densidade de tráfego. O algoritmo 2 formaliza a estratégia DL, que recebe como parâmetros a malha viária $(\mathrm{M})$, o conjunto de veículos $(\mathrm{V})$, o tempo máximo "entre-contatos" $(\tau)$, e as velocidades de download $\left(v_{d}\right)$ e de consumo de dados dentro dos veículos $\left(v_{c}\right)$.

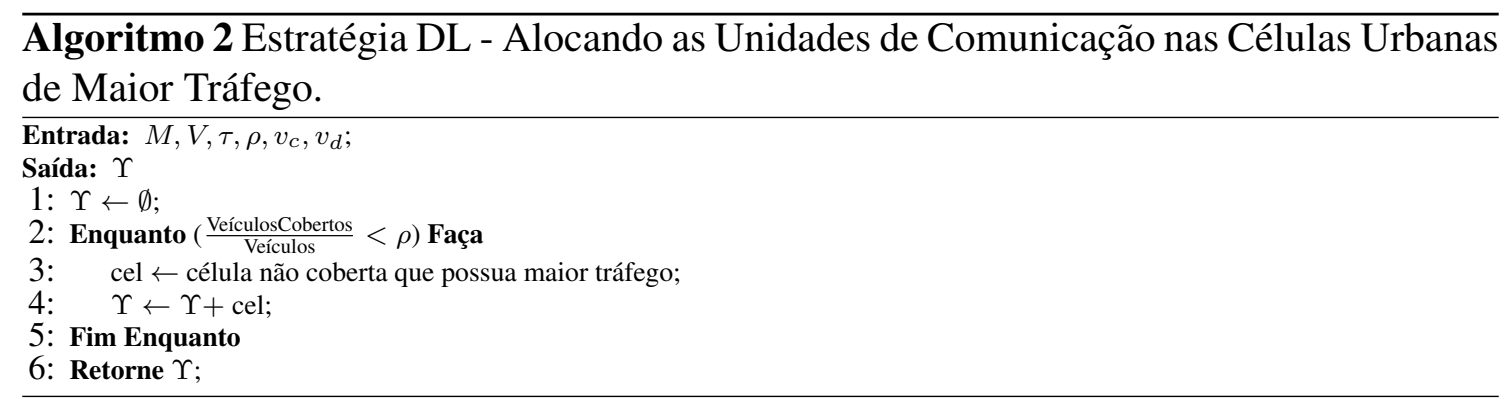

\section{Resultados Experimentais}

Esta seção apresenta os resultados experimentais obtidos. Utiliza-se o trace realístico de mobilidade da cidade de Colônia (Alemanha). O fluxo conta com 7.200 segundos de tráfego e 75.515 veículos. Particiona-se a cidade de Colônia em uma grade de 100 x 100 
células urbanas gerando células com dimensão de $270 \mathrm{~m} x 260 \mathrm{~m}$, uma área com potencial de ser coberta por apenas uma unidade de comunicação (RSU), conforme aponta os resultados reportados em [Teixeira et al., 2014].

Os experimentos são realizados utilizando o simulador $\mathrm{SUMO}^{2}$. Para comparar o desempenho das duas estratégias, dois resultados requerem maior atenção: o investimento necessário para se montar a rede veicular e a cobertura oferecida pela rede. $\mathrm{O}$ investimento pode ser estimado através do número de unidades de comunicação que precisam ser implantadas, dado um nível pretendido de QoS e cobertura. Já a cobertura pode ser analisada através da conectividade provida e da aplicação de métricas de desempenho.

\subsection{Análise de Investimentos}

Aqui busca-se estimar o custo de montagem das redes DL e Sigma- $N$ tendo como base a quantidade de unidades de comunicação que precisam ser implantadas para se atingir uma dada meta de Cobertura Sigma. O primeiro estudo apresenta a quantidade de malha viária que precisa ser coberta para se atingir metas de Cobertura Sigma através dos gráficos 3(a) até 3(d). Neles é possível verificar o percentual da malha viária que precisa ser coberta para se atingir um dado nível de desempenho da rede em termos de veículos experimentando a Cobertura Sigma. Comparando-se ambos os gráficos, nota-se que uma maior relação $r=\frac{\text { velocidade de download }}{\text { velocidade de consumo }}$ reduz a quantidade de RSUs necessárias.

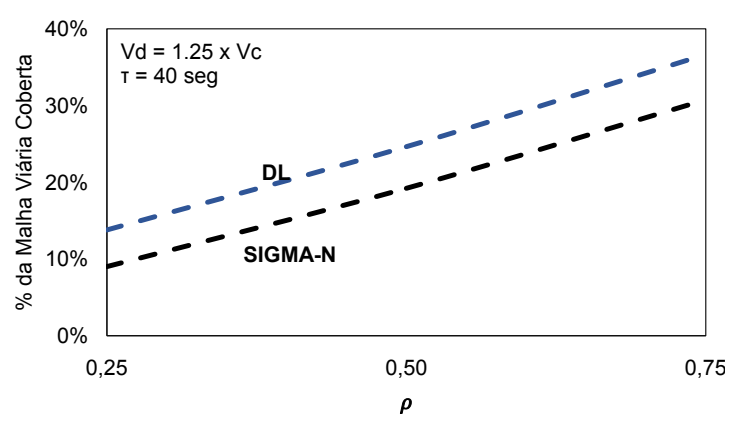

(a) $v_{d}=1,25 \times v_{c}, \tau=40 \mathrm{~s}$

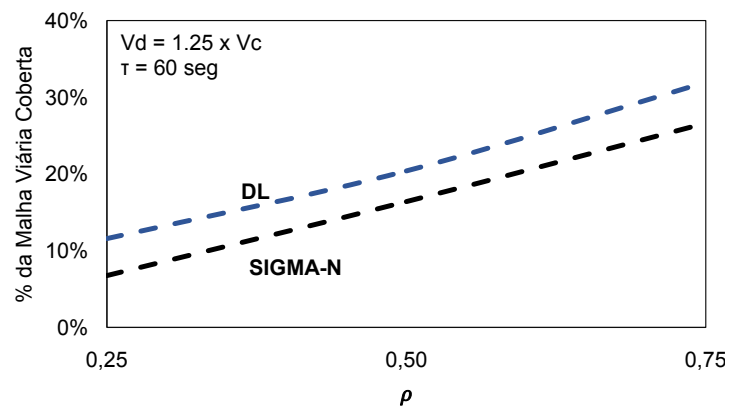

(c) $v_{d}=1,25 \times v_{c}, \tau=60 \mathrm{~s}$

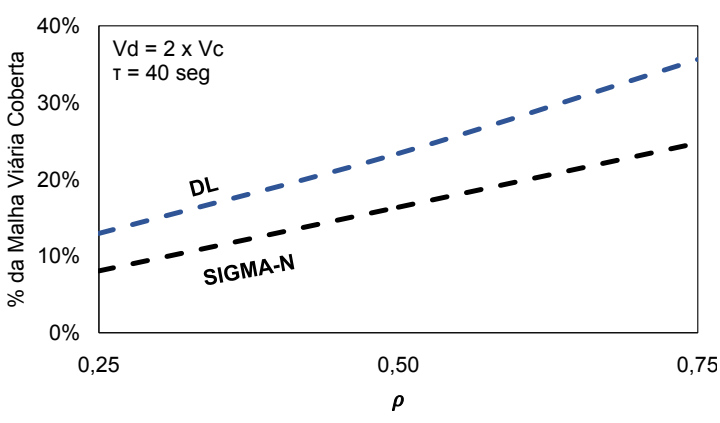

(b) $v_{d}=2 \times v_{c}, \tau=40 \mathrm{~s}$

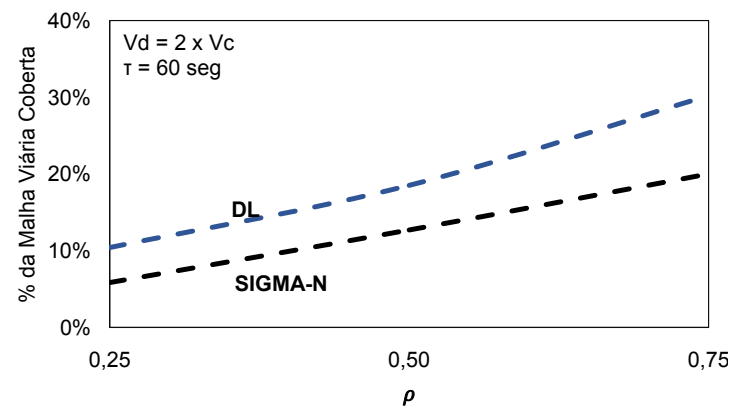

(d) $v_{d}=2 \times v_{c}, \tau=60 \mathrm{~s}$

Figura 3. Os gráficos apresentam a quantidade de malha viária que precisa ser coberta por Sigma-N e DL . O eixo $x$ apresenta $\rho$. O eixo $y$ apresenta a porcentagem coberta da malha viária. Os gráficos (a) e (b) consideram $\tau=40 \mathrm{~s}$. Os gráficos $(\mathbf{c}) \mathbf{e}(\mathbf{d})$ consideram $\tau=60 \mathrm{~s}$.

\footnotetext{
${ }^{2}$ Simulator Sumo: http://sumo-sim.org.
} 
Com o intuito de complementar esse estudo, apresenta-se a redução de investimentos ao se usar a estratégia Sigma- $N$ em relação à alocar as unidades de comunicação nos locais de maior fluxo da malha viária. A figura 4(a) considera o cenário onde a velocidade de download é 1,25 vezes a velocidade de consumo de dados nos veículos. Já a figura 4(b) apresenta o cenário onde a velocidade de download é 2 vezes a velocidade de consumo. Para ambos os cenários, apresenta-se a redução quando considera-se um tempo máximo entre contatos de veículos e infraestrutura de $\tau=40 \mathrm{~s}$ e $\tau=60 \mathrm{~s}$.

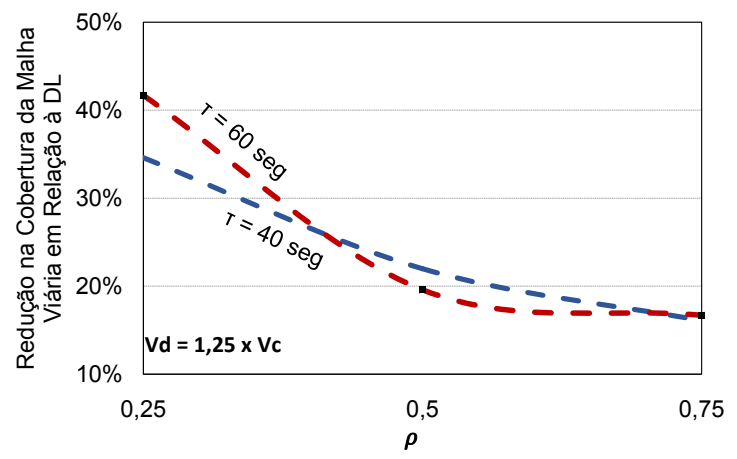

(a) $v_{d}=1.25 \times v_{c}$

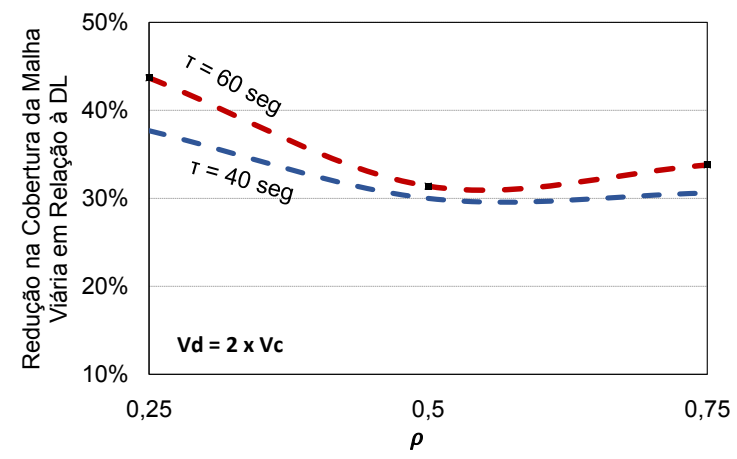

(b) $v_{d}=2 \times v_{c}$

Figura 4. Os gráficos apresentam a redução na quantidade de malha viária que precisa ser coberta ao se optar por Sigma-N em detrimento de DL. 0 eixo $x$ representa a fração de veículos recebendo Cobertura Sigma $(\rho)$, enquanto que o eixo $y$ representa a redução percentual da malha viária que recebeu cobertura.

Nesses gráficos, o eixo $x$ indica a fração de veículos que deve receber Cobertura Sigma $(\rho)$, ou seja, a fração de veículos que deve ser capaz de realizar toda sua viagem sempre tendo carga positiva (streaming em buffer). O gráfico 4(a) indica que, para $\rho$ igual à 0.25 (25\% dos veículos capazes de realizar a viagem sempre com carga positiva), a estratégia Sigma- $N$ reduz a quantidade de malha viária coberta em 35\% quando $\tau=40 \mathrm{~s}$.

Quando $\tau=60 s$, a redução de área coberta é de $42 \%$. Quando considera-se que $75 \%$ dos veículos devem receber Cobertura Sigma, a diferença entre Sigma- $N$ e DL reduz para perto de $20 \%$ de economia. Isso ocorre pois essa fração tão alta de veículos acaba por demandar uma cobertura massiva da malha viária. Raciocínio similar aplica-se ao gráfico 4(b). Em todos os casos analisados, Sigma- $N$ foi capaz de prover a mesma Cobertura Sigma que DL para os parâmetros desejados, porém reduzindo a quantidade de RSUs necessárias, o que implica na redução de investimentos necessários à rede.

\subsection{Análise de Cobertura}

A avaliação da cobertura de rede se dará com base na métrica Rede Delta apresentada em [Silva and Meira Jr, 2015b]. A Rede Delta $\left(\Delta_{\rho_{2}}^{\rho_{1}}\right)$ é uma métrica projetada para avaliar a conectividade em redes veiculares, onde $\rho_{1}$ e $\rho_{2}$ são parâmetros de entrada definindo garantias mínimas de desempenho da rede veicular. O parâmetro $\rho_{1}$ é chamado de fator de duração da conexão e indica a percentagem de tempo que um veículo permanece conectado em relação ao seu tempo total de viagem.

Como exemplo, se o projetista de rede deseja que veículos permaneçam conectados durante $10 \%$ da viagem, $\rho_{1}$ deve ser igual a 0,1 . Já o parâmetro $\rho_{2}$ indica a proporção 
de veículos que possuem o fator de duração de conexão $\rho_{1}$. Assim, uma dada rede veicular é $\Delta_{\rho_{2}}^{\rho_{1}}$ quando $\rho_{2}$ dos veículos permanecem conectados por, pelo menos, $\rho_{1}$ do tempo de duração da viagem.

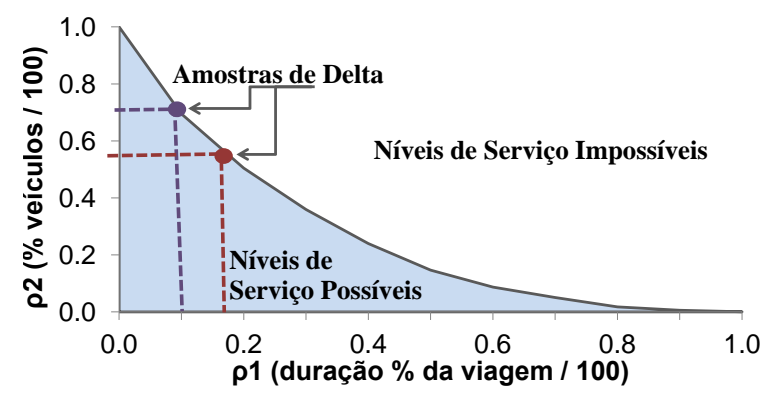

Figura 5. Semicurva indicando a Rede Delta como a relação entre cobertura e fração de veículos.

A figura 5 ilustra a métrica Rede Delta. Diferentemente de outras abordagens, a métrica não é representada por um único valor, mas sim por uma semicurva num plano bidimensional onde o eixo $x$ indica o percentual da viagem que o veículo está coberto $\left(\rho_{1}\right)$ e o eixo $y$ indica a fração de veículos cobertos $\left(\rho_{2}\right)$. Assim, Delta representa a relação entre cobertura e quantidade de veículos. A área sombreada indica níveis de serviço passíveis de serem atingidos pela rede veicular. A semicurva indica o máximo desempenho possível para a rede. A representação de Delta como uma relação entre cobertura e quantidade de veículos tem a vantagem de delimitar completamente a região de operação da rede veicular, permitindo a comparação de redes distintas.

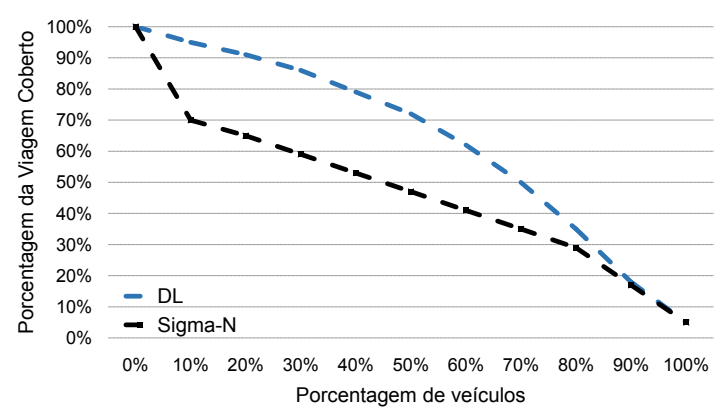

(a) $v_{d}=1,25 \times v_{c}, \tau=40 \mathrm{~s}, \rho=25 \%$

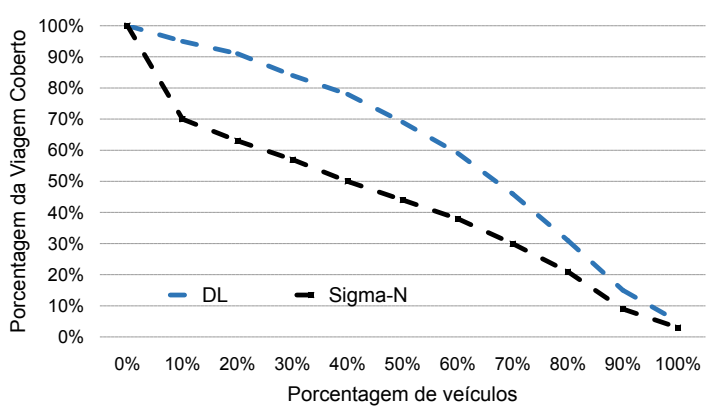

(b) $v_{d}=2 \times v_{c}, \tau=40 \mathrm{~s}, \rho=25 \%$

Figura 6. Os gráficos acima apresentam a Rede Delta para Sigma- $N$ e DL para $\rho=25 \%$ e $\tau=40$ s. $O$ eixo $y$ apresenta a fração de tempo que o veículo está coberto durante toda sua viagem, enquanto que o eixo $x$ apresenta quantos veículos recebem essa cobertura.

As figuras 6(a)-6(b) apresentam a Rede Delta para Sigma-N e DL. A figura (a) considera a velocidade de download igual à 1,25 vezes a velocidade de consumo de dados. O parâmetro $\rho$ é mantido em $25 \%$, enquanto que o tempo entre-contatos $\tau=40 \mathrm{~s}$. Percebe-se que a curva de DL está superior à curva de Sigma-N indicando que DL sempre apresenta uma fração maior de veículos recebendo uma dada cobertura. Ou seja, DL apresenta mais oportunidades de contato entre veículos e a infraestrutura.

O cenário onde $\tau=60 \mathrm{~s}$ é apresentado nas figuras 7(a)-7(b), mantidas constantes todas as demais configurações das figuras 6(a)-6(b). De forma geral, pode-se perceber que 


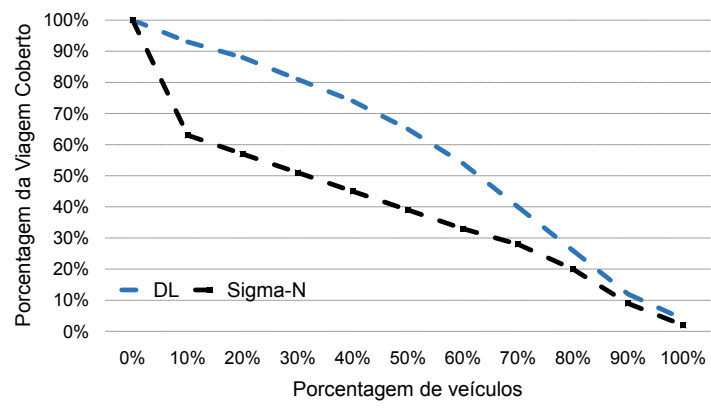

(a) $v_{d}=1,25 \times v_{c}, \tau=60 \mathrm{~s}, \rho=25 \%$

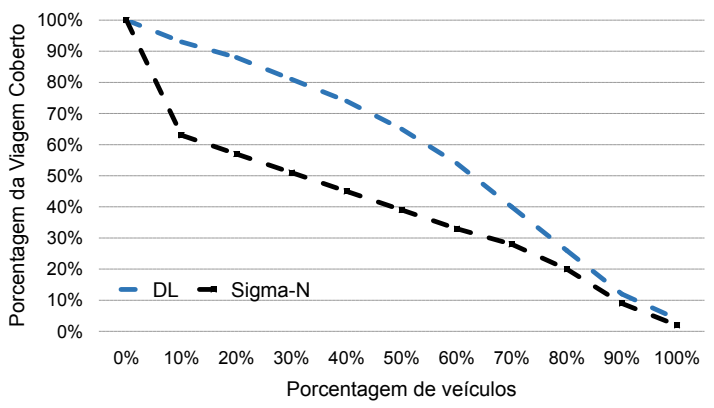

(b) $v_{d}=2 \times v_{c}, \tau=60 \mathrm{~s}, \rho=25 \%$

Figura 7. Os gráficos acima apresentam a Rede Delta para Sigma-N e DL para $\rho=25 \%$ e $\tau=60$ s. $O$ eixo $y$ apresenta a fração de tempo que o veículo está coberto durante toda sua viagem, enquanto que 0 eixo $x$ apresenta quantos veículos recebem essa cobertura.

a curva de DL está sempre acima da curva Sigma- $N$. Isso indica que a rede DL oferece mais oportunidades de contatos entre veículos e a infraestrutura, promovendo, então, uma melhor qualidade de serviço. Visto que DL instala as unidades de comunicação nos locais de maior fluxo, espera-se que veículos trafegando nessas rotas populares sejam sempre capazes de encontrar conectividade. Além disso, a rede DL demanda mais unidades de comunicação ao longo da malha viária. Esse comportamento é similar para todos os cenários estudados.

O lado negativo de DL relaciona-se ao fato da conectividade ser restrita a veículos trafegando somente nas áreas mais populares, segregando veículos que trafegam à margem dessas rotas, podendo inviabilizar o atendimento de veículos fora das rotas populares.

\section{Conclusão}

O presente trabalho apresenta a estratégia de deposição de infraestrutura para redes veiculares Sigma-N. O objetivo central é planejar a infraestrutura de comunicação de forma a realizar a disseminação de streaming da infraestrutura para veículos. Considera-se a velocidade de download de dados da infraestrutura para veículos, bem como a velocidade de consumo de dados dentro do veículo de forma a se realizar uma distribuição que garanta que uma fatia $\rho$ de veículos consiga ter sempre mídia disponível em seu buffer de armazenamento interno.

Compara-se a estratégia proposta com a tática intuitiva de se depositar unidades de comunicação (RSUs) nos locais de maior densidade de veículos. Os resultados demonstram que a aplicação da técnica proposta reduz em (até) $40 \%$ a quantidade de unidades de comunicação que precisam ser depositadas ao longo da malha viária. Por outro lado, a redução de unidades de comunicação implantadas na rede ocasiona uma redução na quantidade de contatos entre veículos e a infraestrutura, algo esperado dado que o baseline sempre implanta as unidades de comunicação nos locais de maior fluxo.

Como trabalhos futuros, o grupo pretende avaliar, também, a incorporação de comunicação veículo-a-veículo para ajudar na disseminação das mídias.

\section{Agradecimentos}

Este trabalho foi parcialmente financiado por recursos do CNPq, CAPES e FAPEMIG. 


\section{Referências}

Aslam, B., Amjad, F., and Zou, C. (2012). Optimal roadside units placement in urban areas for vehicular networks. In Computers and Communications (ISCC), 2012 IEEE Symposium on, pages 000423-000429. IEEE.

Cheng, H., Fei, X., Boukerche, A., Mammeri, A., and Almulla, M. (2013). A geometrybased coverage strategy over urban vanets. In Proceedings of the 10th ACM Symposium on Performance Evaluation of Wireless Ad Hoc, Sensor, \& Ubiquitous Networks, PE-WASUN '13, pages 121-128, New York, NY, USA. ACM.

Chi, J., Jo, Y., Park, H., and Park, S. (2013). Intersection-priority based optimal rsu allocation for vanet. In Ubiquitous and Future Networks (ICUFN), 2013 Fifth International Conference on, pages 350-355.

Deb, K., Pratap, A., Agarwal, S., and Meyarivan, T. (2002). A fast and elitist multiobjective genetic algorithm: Nsga-ii. IEEE transactions on evolutionary computation, 6(2):182-197.

Goldberg, D. E. (1989). Genetic Algorithms in Search, Optimization and Machine Learning. Addison-Wesley Longman Publishing Co., Inc. Boston, MA, USA.

Gossa, J., Janecek, A. G., Hummel, K., Gansterer, W. N., Pierson, J.-M., et al. (2008). Proactive replica placement using mobility prediction. In Mobile Data Management Workshops, 2008. MDMW 2008. Ninth International Conference on, pages 182-189. IEEE.

Konak, A., Coit, D. W., and Smith, A. E. (2006). Multi-objective optimization using genetic algorithms: A tutorial. Rel. Eng. \& Sys. Safety, 91(9):992-1007.

Lee, J. and Kim, C. (2010). A roadside unit placement scheme for vehicular telematics networks. In Kim, T.-h. and Adeli, H., editors, Advances in Computer Science and Information Technology, volume 6059 of Lecture Notes in Computer Science, pages 196-202. Springer Berlin Heidelberg.

Li, Y., Jin, D., Hui, P., and Chen, S. (2015). Contact-aware data replication in roadside unit aided vehicular delay tolerant networks. Mobile Computing, IEEE Transactions on, $\mathrm{PP}(99): 1-1$.

Liu, Y., Niu, J., Ma, J., and Wang, W. (2013). File downloading oriented roadside units deployment for vehicular networks. Journal of Systems Architecture, 59(10, Part B):938 - 946. Advanced Smart Vehicular Communication System and Applications.

Miettinen, K. (1999). Nonlinear multiobjective optimization. Kluwer Academic Publishers, Boston.

Nekoui, M., Eslami, A., and Pishro-Nik, H. (2008). The capacity of vehicular ad hoc networks with infrastructure. In Modeling and Optimization in Mobile, Ad Hoc, and Wireless Networks and Workshops, 2008. WiOPT 2008. 6th International Symposium on, pages 267-272.

Patil, P. and Gokhale, A. (2013). Voronoi-based placement of road-side units to improve dynamic resource management in vehicular ad hoc networks. In Collaboration Technologies and Systems (CTS), 2013 International Conference on, pages 389-396.

Pereira, L., Silva, C. M., and Sarubbi, J. (2017). Planejando a Infraestrutura de Comunicação para a Distribuição de Mídias em Tempo Real para Veículos. In SBRC 2017 (Simpósio Brasileiro de Computação Ubíqua e Pervasiva).

Silva, C. M., Aquino, A. L. L., and Meira Jr, W. (2015). Smart Traffic Light for Low Traffic Conditions. Mobile Networks and Applications, pages 1-9. 
Silva, C. M., Guidoni, D. L., Souza, F. S. H., Pitangui, C. G., Sarubi, J., and Pitsillides, A. (2016a). Gamma Deployment: Designing the Communication Infrastructure in Vehicular Networks Assuring Guarantees on the V2I Inter-Contact Time. In 2016 IEEE 13th International Conference on Mobile Ad Hoc and Sensor Systems (MASS), pages 263-271.

Silva, C. M., Masini, B. M., Ferrari, G., and Thibault, I. (2017a). A Survey on Infrastructure-Based Vehicular Networks. Mobile Information Systems, 2017, Article ID 6123868:28.

Silva, C. M. and Meira Jr, W. (2015a). Design of roadside communication infrastructure with QoS guarantees. In 2015 IEEE Symposium on Computers and Communication (ISCC), pages 439-444.

Silva, C. M. and Meira Jr, W. (2015b). Evaluating the Performance of Heterogeneous Vehicular Networks. In 2015 IEEE 82nd Vehicular Technology Conference (VTC2015-Fall), pages 1-5.

Silva, C. M. and Meira Jr, W. (2015c). Managing Infrastructure-Based Vehicular Networks. In Mobile Data Management (MDM), 2015 16th IEEE International Conference on, volume 2, pages 19-22.

Silva, C. M. and Meira Jr, W. (2016). An architecture integrating stationary and mobile roadside units for providing communication on Intelligent Transportation Systems. In NOMS 2016 - 2016 IEEE/IFIP Network Operations and Management Symposium, pages 358-365.

Silva, C. M., Meira Jr, W., and Sarubbi, J. (2016b). Non-intrusive planning the roadside infrastructure for vehicular networks. IEEE Transactions on Intelligent Transportation Systems, 17(4):938-947.

Silva, C. M., Silva, F. A., Sarubbi, J., Oliveira, T. R., Meira Jr, W., and Nogueira, J. M. S. (2017b). Designing Mobile Content Delivery Networks for the Internet of Vehicles. Vehicular Communications, 8:45 - 55. Internet of Vehicles.

Teixeira, F., Silva, V., Leoni, J., Macedo, D., and Nogueira, J. M. S. (2014). Vehicular networks using the IEEE 802.11p standard: An experimental analysis. Vehicular Communications, 1(2):91 - 96.

Trullols, O., Fiore, M., Casetti, C., Chiasserini, C., and Ordinas, J. B. (2010). Planning roadside infrastructure for information dissemination in intelligent transportation systems. Computer Communications, 33(4):432 - 442.

Van Audenhove, F.-J., Korniichuk, O., Dauby, L., and Pourbaix, J. (2014). The Future of Urban Mobility 2.0: Imperatives to Shape Extended Mobility Ecosystems of Tomorrow.

Xie, B., Xia, G., Chen, Y., and Xu, M. (2013). Roadside infrastructure placement for information dissemination in urban its based on a probabilistic model. In Network and Parallel Computing, volume 8147 of Lecture Notes in Computer Science, pages 322-331. Springer Berlin Heidelberg.

Ye, Q., Ma, C., He, R., Xiao, Q., and Zhang, W. (2015). Multi-objective optimisation for taxi ridesharing route based on non-dominated sorting genetic algorithm. IJWMC, 8(3):262-270.

Zheng, Z., Lu, Z., Sinha, P., and Kumar, S. (2010). Maximizing the contact opportunity for vehicular internet access. In INFOCOM, 2010 Proceedings IEEE, pages 1-9. 